\title{
Strong Dynamical Modulation of the Cooling of the Polar Stratosphere Associated with the Antarctic Ozone Hole
}

\author{
Andrew OrR, Thomas J. Bracegirdle, AND J. SCOTt Hosking \\ British Antarctic Survey, Cambridge, United Kingdom \\ WUHU FENG \\ National Centre for Atmospheric Science, University of Leeds, Leeds, United Kingdom \\ HOWARD K. ROSCOE \\ British Antarctic Survey, Cambridge, United Kingdom \\ JOANNA D. HAIGH \\ Imperial College London, London, United Kingdom
}

(Manuscript received 17 July 2012, in final form 31 October 2012)

\begin{abstract}
A model simulation forced by prescribed ozone depletion shows strong dynamical modulation of the springtime cooling of the polar stratosphere associated with the Antarctic ozone hole. The authors find that in late spring the anomalous radiative cooling in response to ozone depletion is almost canceled above $\sim 100 \mathrm{hPa}$ by an increase in dynamical heating. Between $\sim 300$ and $\sim 100 \mathrm{hPa}$, however, it is enhanced by a reduction in dynamical heating, resulting in the descent of the cold anomaly down to the tropopause. In early summer increased dynamical heating dominates as the radiative cooling diminishes so that the cold anomaly associated with the delayed breakup of the stratospheric vortex is reduced. The anomalous dynamical heating is driven by changes in the Brewer-Dobson circulation arising primarily from the dissipation of resolved-scale waves. The model changes are broadly consistent with trends from reanalysis and offline diagnoses of heating rates using a radiation scheme. These results help one to understand dynamically induced change in the evolution and timing of the stratospheric vortex in recent decades and will help to enable improved simulation of the Southern Hemisphere climate.
\end{abstract}

\section{Introduction}

In recent decades, the temperature of the Southern Hemisphere polar stratosphere has undergone pronounced changes, as observed in radiosonde records over Antarctica for levels up to $\sim 30 \mathrm{hPa}$. The data show substantial cooling in the spring stratosphere above $300 \mathrm{hPa}$ since about 1985 with maximum cooling of $\sim 10 \mathrm{~K}$ in the lower stratosphere $(\sim 100 \mathrm{hPa})$ in November (Randel and Wu 1999). Smaller magnitude cooling extends throughout summer. The cooling increases the

Corresponding author address: Andrew Orr, British Antarctic Survey Climate Programme, High Cross, Madingley Road, Cambridge CB3 0ET, United Kingdom.

E-mail: anmcr@bas.ac.uk latitudinal temperature gradient, resulting in a strengthening of the stratospheric vortex (Thompson and Solomon 2002), as well as a subsequent delay in its springtime breakup (Haigh and Roscoe 2009).

The dominant cause for these temperature trends is the springtime Antarctic ozone hole (Gillett and Thompson 2003), which extends from around 150 to $30 \mathrm{hPa}$, with maximum ozone losses exceeding $90 \%$ at $70 \mathrm{hPa}$ around the late 1990s (Solomon et al. 2005). Although it is clear that ozone loss has a direct impact on the stratosphere by means of radiative cooling, a number of modeling studies (e.g., Christiansen et al. 1997; Manzini et al. 2003; Li et al. 2010; McLandress et al. 2010; Orr et al. 2012) suggest that it can also lead to changes to dynamical heating of the polar stratosphere, which occurs when air associated with the downwelling part of the wave-driven 
Brewer-Dobson circulation is compressed and adiabatically heated. In a previous study (Orr et al. 2012), the authors used momentum budget analysis within the transformed Eulerian mean (TEM) framework (e.g., Dunkerton et al. 1981) to show that the changes in the zonally averaged circulation associated with the ozone hole were consistent with dynamical forcing changes. In this paper, we determine the contributions of radiative and dynamical processes to the associated temperature changes. Our results are important to explain the timing and evolution of the Southern Hemisphere stratospheric vortex in recent decades and to help understand why most current climate models exhibit a later than observed breakup of the vortex (e.g., Gillett and Thompson 2003; Eyring et al. 2006).

\section{Method of analysis, model, and reanalysis dataset}

\section{a. Method of analysis}

The TEM formulation connects the dynamical heating rate of the zonally averaged potential temperature $\bar{Q}_{d}^{\theta}$ to the wave-driven Brewer-Dobson circulation via the (ageostrophic) equation (Dunkerton et al. 1981)

$\bar{Q}_{d}^{\theta}=-\rho_{0}^{-1}\left\{\rho_{0}\left[\left(\frac{\bar{\theta}_{\phi}}{a \bar{\theta}_{z}}\right) \overline{v^{\prime} \theta^{\prime}}+\overline{w^{\prime} \theta^{\prime}}\right]\right\}_{z}-\frac{1}{a} \bar{\theta}_{\phi} \bar{v}^{*}-\bar{\theta}_{z} \bar{w}^{*}$,

where $\theta$ is potential temperature, $\rho_{0}$ is the background density, $a$ is the radius of the earth, $v$ is the meridional wind component, $w$ is the vertical wind component, $\left(\bar{v}^{*}, \bar{w}^{*}\right)$ are the meridional and vertical components of the residual circulation (which in the TEM formulation approximates the Brewer-Dobson circulation), $z$ is the vertical coordinate $[=-H \ln (p / 1000)$ in which $H$ is a scale height $(6.4 \mathrm{~km})$ and $p$ is pressure $(\mathrm{hPa})], \phi$ is latitude, subscripts $z$ and $\phi$ denote the partial derivatives with respect to height and latitude, overbars denote zonal averages, and primes denote deviations from zonal averages. The dominant term on the rhs of (1) is from $-\bar{\theta}_{z} \bar{w}^{*}$, which represents dynamical heating from compression. We compute the zonally averaged dynamical heating rate of the actual temperature $\bar{Q}_{d}$ by using the relationship $\bar{Q}_{d}=\bar{Q}_{d}^{\theta}\left(p / p_{s}\right)^{R / c_{p}}$ in which $R$ is the gas constant of air and $c_{p}$ is the specific heat capacity at constant pressure. Following this, the zonally averaged thermal balance of the atmosphere can be expressed as

$$
\bar{T}_{t}=\bar{Q}_{r}+\bar{Q}_{d}+\varepsilon,
$$

where $\bar{T}_{t}$ is temperature tendency, $\bar{Q}_{r}$ is the radiative heating rate, and $\varepsilon$ is the residual heating rate that would be produced by unresolved smaller-scale forcing (e.g., due to gravity wave drag, which must be parameterized; e.g., Orr et al. 2010).

\section{b. Model}

The model simulations are those of Orr et al. (2012). The runs are described in detail in this work. The model is the atmosphere-only component of the Hadley Centre Global Environmental Model, version 3 (HadGEM3-A) (Martin et al. 2006). The horizontal resolution is $1.25^{\circ} \times$ $1.875^{\circ}$ with 85 vertical levels extending from the surface to $\sim 85 \mathrm{~km}$. Following $6 \mathrm{yr}$ of spinup, a control (perturbed) simulation was run for $24 \mathrm{yr}$ forced with a seasonally varying ozone distribution representative of the pre-ozone-hole (ozone hole) period. Both simulations were run with the same climatological sea surface temperatures. The temperature response is evaluated by examining mean differences between the two simulations. Significance is tested using a one-sided Student's $t$ test (assuming 24 degrees of freedom).

\section{c. Reanalysis dataset}

The reanalysis dataset is the 40-yr European Centre for Medium-Range Weather Forecasts Re-Analysis (ERA-40) (Uppala et al. 2005) and the impact of ozone depletion on temperature change is evaluated by examining 23-yr linear trends (1979-2001) as it has been demonstrated that most of the increase in ozone loss occurred within this period (Huck et al. 2007). Reanalysis trends were computed because the 23 -yr sample size enabled statistically significant changes to be determined. Six-hourly fields of temperature were retrieved at a resolution of $2^{\circ} \times 2^{\circ}$ at 17 vertical pressure levels from 1000 to $10 \mathrm{hPa}$. The $\bar{Q}_{r}$ data are diagnosed using the Single-Layer Isentropic Model of Chemistry And Transport (SLIMCAT) chemical transport model (Chipperfield 2006), as this field is not part of the publicly accessible ERA-40 dataset. SLIMCAT has a resolution of $5.6^{\circ} \times 5.6^{\circ}$ and 24 levels extending from the surface to $\sim 55 \mathrm{~km}$ and is forced by 6-hourly ERA-40 surface pressure and 60-level winds and temperature. The model includes the National Center for Atmospheric Research Community Climate Model radiation scheme (Briegleb 1992) for the calculation of $\bar{Q}_{r}$ by accounting for the effect of shortwave $\left(\mathrm{O}_{2}, \mathrm{O}_{3}, \mathrm{CO}_{2}\right.$, and $\left.\mathrm{H}_{2} \mathrm{O}\right)$ and longwave $\left(\mathrm{O}_{3}, \mathrm{CO}_{2}, \mathrm{H}_{2} \mathrm{O}, \mathrm{CH}_{4}, \mathrm{~N}_{2} \mathrm{O}, \mathrm{F} 11\right.$, and F12) radiatively active gases specified (with the exception of ozone) from a climatology (Chipperfield 2006). Ozone is calculated in the model by a detailed stratospheric chemistry scheme, which gives a good representation of its observed interannual variability and 


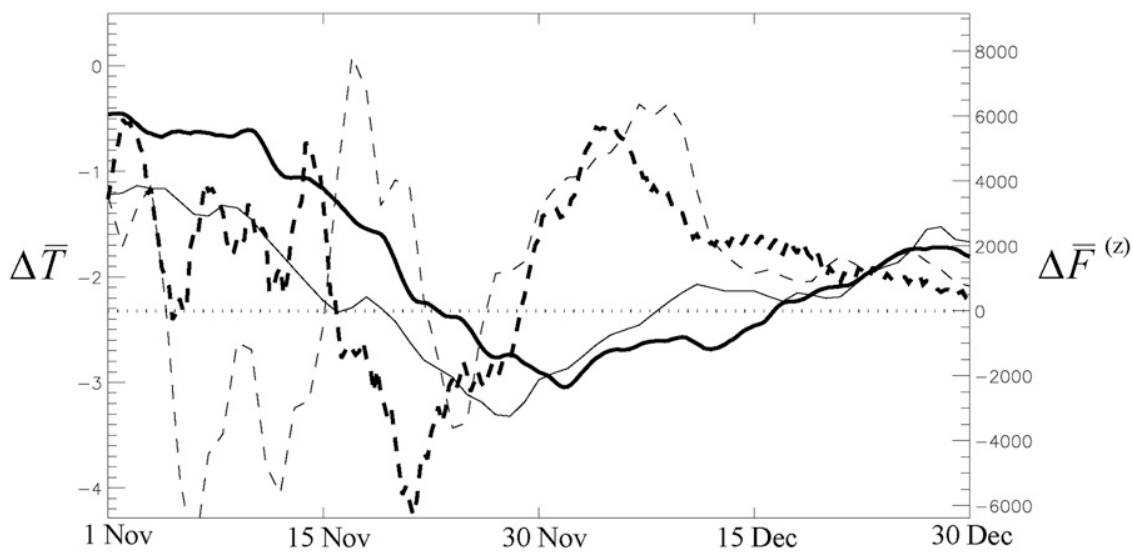

FIG. 1. Simulated (thick lines) and reanalysis (thin lines) daily changes of zonally averaged temperature $\Delta \bar{T}$ averaged from 300 to $30 \mathrm{hPa}$ and from $60^{\circ}$ to $80^{\circ} \mathrm{S}$ (solid lines) and zonally averaged vertical EP flux $\Delta \bar{F}^{(z)}$ at $30 \mathrm{hPa}$ averaged from $40^{\circ}$ to $80^{\circ} \mathrm{S}$ (dashed lines). Simulated changes are the 24-yr averaged differences between the ozone-hole and the pre-ozone-hole model runs. Reanalysis changes are ERA-40 23-yr linear trends (1979-2001). The units for the simulated (reanalysis) changes are $\mathrm{K}\left(\mathrm{K} \mathrm{decade}^{-1}\right)$ for $\Delta \bar{T}$ and $\mathrm{m}^{3} \mathrm{~s}^{-2}\left(\mathrm{~m}^{3} \mathrm{~s}^{-2} \mathrm{decade}^{-1}\right)$ for $\Delta \bar{F}^{(z)}$. Latitudinal (vertical) averages are area (height) weighted.

recent trends (Feng et al. 2005, 2007; WMO 2007). The $\bar{Q}_{d}$ trends computed directly from the reanalysis show spurious changes due to noisy $w$ fields (Wohltmann and Rex 2007), so we compute this as the residual of $\bar{T}_{t}$ and $\bar{Q}_{r}$ trends. Significance is again tested using a one-sided Student's $t$ test (assuming 23 degrees of freedom).

\section{Results}

\section{a. Model differences}

Figure 1 compares the daily evolution for austral late springtime/early summertime (i.e., November-December) between the model difference and reanalysis trend of zonally averaged temperature $\Delta \bar{T}$ averaged from $60^{\circ}$ to $80^{\circ} \mathrm{S}$ and from 300 to $30 \mathrm{hPa}$ (i.e., the region of observed stratospheric cooling). These curves show a marked similarity (although offset by $\sim 5$ days), describing a cooling during most of November with a maximum cooling of $\sim 3 \mathrm{~K}$ and a gradual erosion of the anomalously cold temperatures during December. These model temperature differences were displayed by Orr et al. (2012, their Fig. 2), which presented the results as four 15-day averages encompassing the onset (2-16 November), growth (17 November-1 December), decline (2-16 December), and decay (17 December-1 January) of the cooling response in the polar stratosphere. To quantify the contributions made by dynamical and radiative changes, Fig. 2 here shows model differences of the heating rate components (defined as $\Delta \bar{T}_{t}, \Delta \bar{Q}_{r}$, and $\Delta \bar{Q}_{d}$ ) averaged over the same four stages. The similarity between the daily evolution of the model and reanalysis temperature changes suggests that the model differences presented in Fig. 2 represent the actual seasonal evolution of the temperature response associated with the ozone hole.

The TEM formulation is strictly regarded as being applicable to disturbances of the zonally averaged circulation that evolve over sufficiently long time scales for the condition of quasi steadiness to be approached (Haynes et al. 1991). Despite this, the methodology has been demonstrated to be suitable for studying relatively transient events in the stratosphere such as sudden stratospheric warmings (e.g., Dunkerton et al. 1981; Palmer 1981). Similarly, we argue that this methodology is suitable here due to the use of 15-day averaging periods and differences. In Fig. 2 this is particularly evident during the onset and growth stages, which show quasi steadiness in that $\Delta \bar{T}_{t}$ is composed of rather small differences between the two large and opposing $\Delta \bar{Q}_{r}$ and $\Delta \bar{Q}_{d}$ terms. We note that this is not the case during the decline stage, but our confidence in the suitability of the TEM methodology is strengthened by the general good agreement between $\Delta \bar{T}_{t}$ and the net heating rate $\left(\Delta \bar{Q}_{r}+\Delta \bar{Q}_{d}\right)$.

During the onset stage, $\Delta \bar{T}_{t}$ shows weak cooling from 200 to $30 \mathrm{hPa}$, with a maximum cooling of $\sim 0.1 \mathrm{~K} \mathrm{day}^{-1}$ from 100 to $70 \mathrm{hPa}$, consistent with the beginning of the anomalously cold temperatures (Fig. 1). The $\Delta \bar{Q}_{r}$ shows much stronger cooling, broadly confined to the same region as the $\Delta \bar{T}_{t}$ cooling, which peaks at about $-0.45 \mathrm{~K} \mathrm{day}^{-1}$ near $30 \mathrm{hPa}$. This cooling is largely offset by equally strong $\Delta \bar{Q}_{d}$ warming, although the $\Delta \bar{Q}_{d}$ warming does not extend to as lower altitudes or 

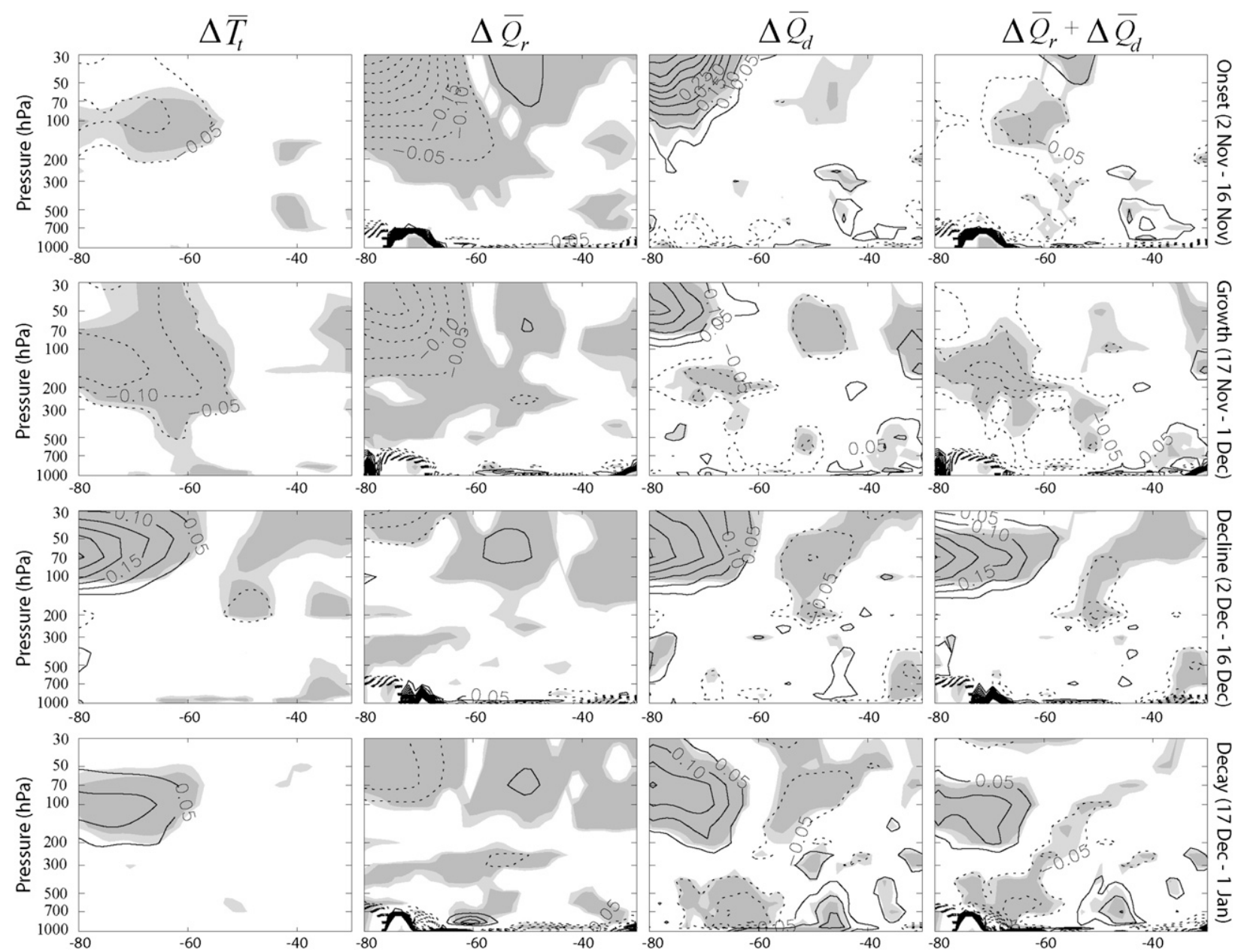

FIG. 2. Latitude-height cross sections of the simulated changes of the zonally averaged terms in Eq. (2) for (top) to (bottom) the onset (2-16 Nov), growth (17 Nov-1 Dec), decline (2-16 Dec) and decay (17 Dec-1 Jan) stages: from (left) to (right) temperature tendency $\Delta \bar{T}_{t}$ and the radiative $\left(\Delta \bar{Q}_{r}\right)$, dynamical $\left(\Delta \bar{Q}_{d}\right)$ and net $\left(\Delta \bar{Q}_{r}+\Delta \bar{Q}_{d}\right)$ heating rates. Simulated changes are the 24-yr-averaged differences between the ozone-hole and pre-ozone-hole model runs. The contour interval is $0.05 \mathrm{~K} \mathrm{day}^{-1}$; dashed lines indicate negative values, and the zero contour is omitted; shading denotes regions where the $p$ values of the differences are $\leq 0.1$ (light shading) and $\leq 0.05$ (dark shading). Latitudinal averages are area weighted.

latitudes as the $\Delta \bar{Q}_{r}$ cooling. Consequently, the net heating rate shows a weak cooling between $60^{\circ}$ and $70^{\circ} \mathrm{S}$, which agrees in amplitude with $\Delta \bar{T}_{t}$. However, at higher latitudes the net heating rate underestimates $\Delta \bar{T}_{t}$ by about $-0.05 \mathrm{~K} \mathrm{day}^{-1}$ owing to the almost exact cancellation in this region between $\Delta \bar{Q}_{r}$ and $\Delta \bar{Q}_{d}$.

During the growth stage, the $\Delta \bar{T}_{t}$ cooling increases and extends downward toward the tropopause $(\sim 300 \mathrm{hPa})$ relative to the onset stage, with a maximum cooling of $\sim 0.15 \mathrm{~K} \mathrm{day}^{-1}$ from 200 to $70 \mathrm{hPa}$, consistent with the strengthening of the anomalously cold temperatures (Fig. 1). The $\Delta \bar{Q}_{r}$ cooling reduces in magnitude (maximum cooling of $\sim 0.25 \mathrm{~K} \mathrm{day}^{-1}$ near $50 \mathrm{hPa}$ ) and latitudinal extent but broadly maintains the same vertical extent relative to the onset stage. Again, this cooling is largely offset by equally strong, but comparatively less extensive, $\Delta \bar{Q}_{d}$ warming. However, it is $\Delta \bar{Q}_{d}$ and not $\Delta \bar{Q}_{r}$ that shows moderate cooling of $\sim 0.15 \mathrm{~K} \mathrm{day}^{-1}$ from 300 to $150 \mathrm{hPa}$ : this is below the altitude where most of the ozone loss occurs, so $\Delta \bar{Q}_{r}$ is close to zero here. Consequently, the net heating rate shows weak cooling throughout the stratosphere, peaking at about $-0.1 \mathrm{~K} \mathrm{day}^{-1}$ from 200 to $100 \mathrm{hPa}$. Although this has the same sign as the $\Delta \bar{T}_{t}$ cooling, it underestimates its amplitude by about $-0.1 \mathrm{~K} \mathrm{day}^{-1}$ between 70 and $80^{\circ} \mathrm{S}$ and by about $-0.05 \mathrm{~K} \mathrm{day}^{-1}$ at $60^{\circ} \mathrm{S}$.

During the decline stage, $\Delta \bar{T}_{t}$ from 150 to $30 \mathrm{hPa}$ reverses in sign relative to the growth stage and shows substantial warming, with a peak of $\sim 0.3 \mathrm{~K} \mathrm{day}^{-1}$ at $70 \mathrm{hPa}$, consistent with the beginning of the erosion of the anomalously cold temperatures (Fig. 1). The $\Delta \bar{Q}_{r}$ 
cooling decreases in both magnitude and depth relative to the growth stage, peaking at about $-0.15 \mathrm{~K} \mathrm{day}^{-1}$ at $30 \mathrm{hPa}$. In contrast, the $\Delta \bar{Q}_{d}$ warming, which peaks at $\sim 0.3 \mathrm{~K} \mathrm{day}^{-1}$ at $70 \mathrm{hPa}$, has strengthened slightly. Consequently, the $\Delta \bar{Q}_{d}$ warming is only partially canceled by $\Delta \bar{Q}_{r}$ cooling so that the net heating rate shows substantial warming from 150 to $30 \mathrm{hPa}$ with a peak of $\sim 0.3 \mathrm{~K} \mathrm{day}^{-1}$ at $70 \mathrm{hPa}$, in agreement with $\Delta \bar{T}_{t}$.

During the decay stage, the $\Delta \bar{T}_{t}$ warming is confined from 200 to $50 \mathrm{hPa}$, with a peak of $\sim 0.1 \mathrm{~K} \mathrm{day}^{-1}$ at $100 \mathrm{hPa}$, consistent with weaker erosion of the anomalously cold temperatures (Fig. 1). Both $\Delta \bar{Q}_{r}$ cooling and $\Delta \bar{Q}_{d}$ warming have decreased in magnitude relative to the decline stage. The $\Delta \bar{Q}_{d}$ warming rate, however, still exceeds the cooling rate so that the net heating rate is positive with a peak of $\sim 0.1 \mathrm{~K} \mathrm{day}^{-1}$ at around $100 \mathrm{hPa}$, in agreement with $\Delta \bar{T}_{t}$.

The $\Delta \bar{Q}_{d}$ warming apparent over the polar regions in each of the four stages coincides with anomalously strong downwelling (cf. Fig. 3 of Orr et al. 2012), that is, a strengthening of the Brewer-Dobson circulation. However, the anomalous eastward wave driving in the lower stratosphere, which is responsible for the strengthening of the stratospheric vortex during the onset and growth stages (cf. Fig. 3 of Orr et al. 2012), would typically be associated with a weakening of the Brewer-Dobson circulation, that is, anomalously weak downwelling. Our results therefore suggest that increased wave driving at levels higher than $30 \mathrm{hPa}$ is important, which following the "downward control principle" would control the flow across this level (Haynes et al. 1991).

To investigate this further, Fig. 1 also includes the temporal evolution of the model difference of zonally averaged vertical Eliassen-Palm (EP) flux $\Delta \bar{F}^{(z)}$ averaged from $40^{\circ}$ to $80^{\circ} \mathrm{S}$ at $30 \mathrm{hPa}$, which is equivalent to the difference in total extratropical EP flux divergence $\boldsymbol{\nabla} \cdot \mathbf{F}$ above the 30 -hPa level, that is, the total wave driving at higher levels (see Fusco and Salby 1999). During the onset, decline, and decay stages the increased $\Delta \bar{F}^{(z)}$ (cf. Fig. 3 of Orr et al. 2012) is consistent with the increased polar downwelling and associated increased dynamical heating (Fig. 2). The increase in wave driving can be explained by increased critical level filtering in response to the stronger vortex winds (as suggested by Manzini et al. 2003). During the growth stage the increased downwelling is being driven despite an overall small decrease in $\Delta \bar{F}^{(z)}$. However, separating $\bar{w}^{*}\left\{=\bar{w}+(a \cos \phi)^{-1} \partial\left[\cos \phi\left(\overline{v^{\prime} \theta^{\prime} / \bar{\theta}_{z}}\right)\right] / \partial \phi\right\}$ into its $\bar{w}$ component and the term involving the eddy heat flux $\overline{v^{\prime} \theta^{\prime}}$, which is directly related to the vertical EP flux, reveals that the differences in $\bar{w}^{*}$ during this stage are driven less by waves than by changes in $\bar{w}$ (not shown).

\section{b. Reanalysis trends}

Figure 3 is as Fig. 2 but shows trends from reanalysis/ SLIMCAT. Note the same notation is used to describe the trends (which are expressed as the change per decade) as was used above to describe the model differences, that is, $\Delta \bar{T}_{t}, \Delta \bar{Q}_{r}$, and $\Delta \bar{Q}_{d}$. There is a broad similarity to the model differences, including (i) during the growth stages (and to a lesser extent the onset stage) some of the $\Delta \bar{Q}_{r}$ cooling above $\sim 200 \mathrm{hPa}$ is canceled by increased $\Delta \bar{Q}_{d}$ heating; (ii) during the growth stage the $\Delta \bar{T}_{t}$ cooling increases and extends downward toward the tropopause relative to the onset stage, caused by decreased $\Delta \bar{Q}_{d}$ heating; and (iii) during the decline and decay stages $\Delta \bar{T}_{t}$ reverses to warming, caused by increased $\Delta \bar{Q}_{d}$ heating overwhelming the $\Delta \bar{Q}_{r}$ cooling. However, one notable difference is that the $\Delta \bar{Q}_{r}$ cooling does not appear to diminish in early summer during the decline and decay stages. Some aspects of the reanalysis trend in $\Delta \bar{F}^{(z)}$ are also broadly consistent with the model differences (Fig. 1), particularly during the decline and decay stages that are similarly preceded by a sharp increase in $\Delta \bar{F}^{(z)}$ resulting in an increase in wave driving. However, it is noted that the reanalysis trend shows a decrease (increase) in $\Delta \bar{F}^{(z)}$ during the onset (growth) stages opposite to that of the model differences.

\section{Conclusions}

Our analysis clarifies the importance of dynamical heating in modulating recent stratospheric temperature trends within the Antarctic ozone hole. First, in late spring much of the radiative cooling above $\sim 200 \mathrm{hPa}$ is canceled by increased dynamical heating; that is, the actual cooling is due to subtle differences between two large opposing terms. Second, in late spring the radiative cooling is enhanced by a decrease in dynamical heating between $\sim 300$ and $\sim 100 \mathrm{hPa}$; that is, changes to dynamical heating drive the migration of cooling downward to the tropopause. Third, during early summer the radiative cooling is overwhelmed by increased dynamical heating, resulting in the erosion of the anomalously cold temperatures. These results suggest that there will be subtle changes in the temporal evolution of the thermal balance as one or other term undergoes modest changes from year to year as the century progresses.

The lack of balance between the model difference in actual temperature tendency and the net heating rate during the onset and growth stages suggests that some additional dynamical heating is required - that is, additional wave drag. This may be partly due to ignoring the effects of unresolved gravity wave drag in Eq. (2) as the model difference fields showed an increase in parameterized gravity wave drag during November (not shown). 

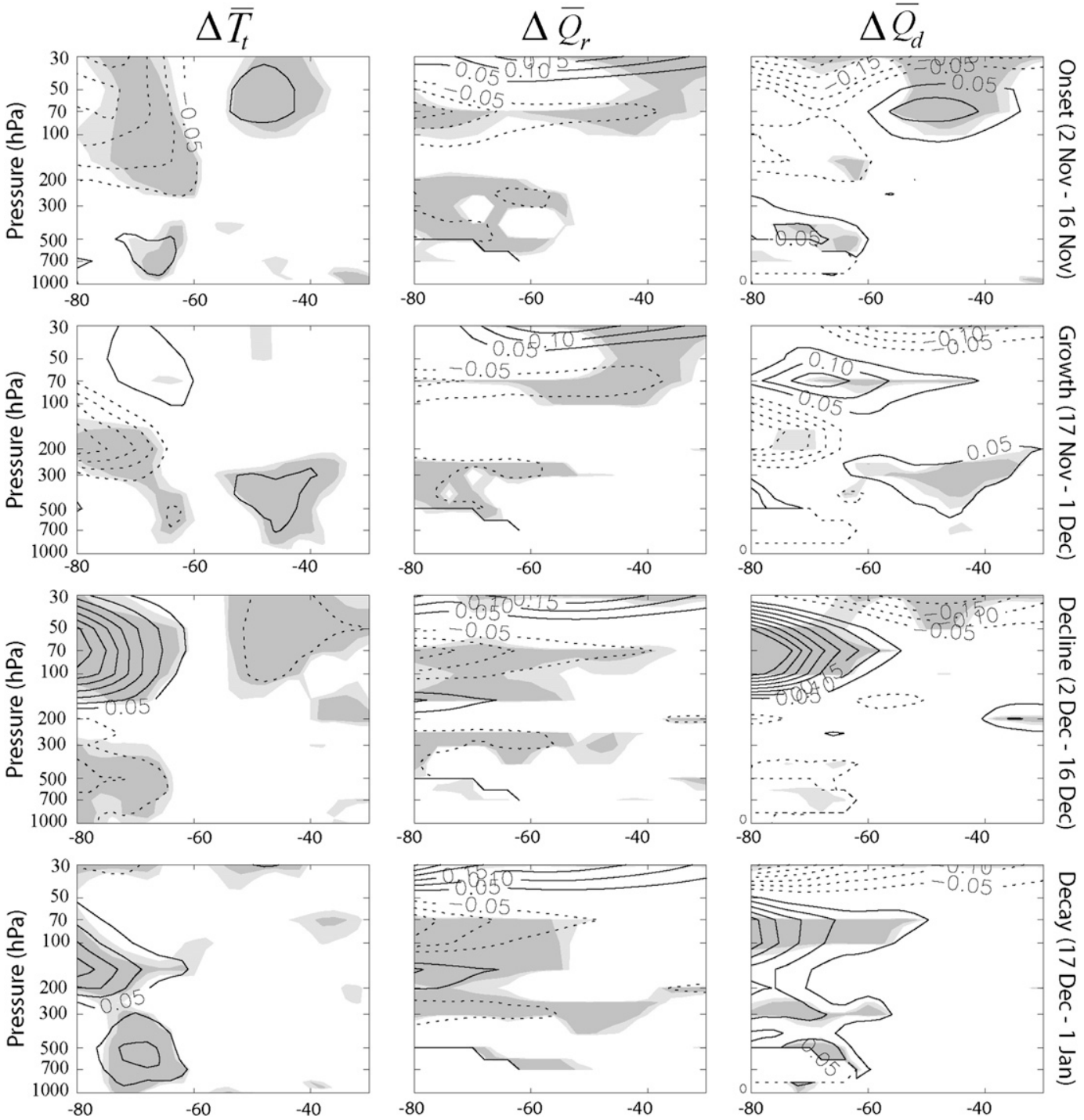

FIG. 3. As in Fig. 2 but showing 23-yr linear trends (1979-2001) of ERA-40 temperature tendency, diagnosed radiative heating rate from SLIMCAT, and dynamical heating rate (computed as the residual). The same notation is used to describe the trends (expressed as the change per decade) as was used to describe the model differences: that is, $\Delta \bar{T}_{t}, \Delta \bar{Q}_{r}$, and $\Delta \bar{Q}_{d}$. Note that, by defining $\Delta \bar{Q}_{d}$ as the residual, the trend in net heating rate $\left(\Delta \bar{Q}_{r}+\Delta \bar{Q}_{d}\right)$ equals $\Delta \bar{T}_{t}$ and so is not shown. The contour interval is $0.05 \mathrm{~K} \mathrm{day}^{-1} \mathrm{decade}^{-1}$.

Acknowledgments. The comments of the two anonymous reviewers contributed considerably to the improvement of the original manuscript. Thanks are given to P. Braesicke, M. Chipperfield, P. Haynes, and M. McIntyre for useful discussions and to L. Abraham, S. Hardiman, S. Osprey, and P. Telford for helping create the ozone climatologies.

\section{REFERENCES}

Briegleb, B. P., 1992: Delta-Eddington approximation for solar radiation in the NCAR Community Climate Model. J. Geophys. Res., 97, 7603-7612.
Chipperfield, M. P., 2006: New version of the TOMCAT/SLIMCAT off-line chemical transport model: Intercomparison of stratospheric tracer experiments. Quart. J. Roy. Meteor. Soc., 132, 1179-1203.

Christiansen, B., A. Guldberg, A. W. Hansen, and L. P. Rishøjgaard, 1997: On the response of a three-dimensional general circulation model to imposed changes in ozone distribution. J. Geophys. Res., 102, 13 051-13 077.

Dunkerton, T., C.-P. F. Hsu, and M. E. McIntyre, 1981: Some Eulerian and Lagrangian diagnostics for a model stratospheric warming. J. Atmos. Sci., 38, 819-843.

Eyring, V., and Coauthors, 2006: Assessment of temperature, trace species, and ozone in chemistry-climate model simulations of the recent past. J. Geophys. Res., 111, D22308, doi:10.1029/ 2006JD007327. 
Feng, W., and Coauthors, 2005: Three-dimensional model study of the Arctic ozone loss in 2002/03 and comparison with 1999/ 2000 and 2003/04. Atmos. Chem. Phys., 5, 139-152.

- M. P. Chipperfield, M. Dorf, K. Pfeilsticker, and P. Ricaud, 2007: Mid-latitude ozone changes: Studies with a 3-D CTM forced by ERA-40 analyses. Atmos. Chem. Phys., 7, 2357-2369.

Fusco, A. C., and M. L. Salby, 1999: Interannual variations of total ozone and their relationship to variations of planetary wave activity. J. Climate, 12, 1619-1629.

Gillett, N. P., and D. W. J. Thompson, 2003: Simulation of recent Southern Hemisphere climate change. Science, 302, 273-275.

Haigh, J. D., and H. R. Roscoe, 2009: The final warming date of the Antarctic polar vortex and influences on its interannual variability. J. Climate, 22, 5809-5819.

Haynes, P. H., C. J. Marks, M. E. McIntyre, T. G. Shepherd, and K. P. Shine, 1991: On the "downward control" of extratropical diabatic circulations by eddy-induced mean zonal forces. J. Atmos. Sci., 48, 651-678.

Huck, P. E., S. Tilmes, G. E. Bodeker, W. J. Randel, A. J. McDonald, and H. Nakajima, 2007: An improved measure of ozone depletion in the Antarctic stratosphere. J. Geophys. Res., 112, D11104, doi:10.1029/2006JD007860.

Li, F., P. A. Newman, and R. S. Stolarski, 2010: Relationships between the Brewer-Dobson circulation and the southern annular mode during austral summer in coupled chemistryclimate model simulations. J. Geophys. Res., 115, D15106, doi:10.1029/2009JD012876.

Manzini, E., B. Steil, C. Brühl, M. A. Giorgetta, and K. Kruger, 2003: A new interactive chemistry-climate model: 2. Sensitivity of the middle atmosphere to ozone depletion and increase in greenhouse gases and implications for recent stratospheric cooling. J. Geophys. Res., 108, 4429, doi:10.1029/ 2002JD002977.

Martin, G. M., M. A. Ringer, V. D. Pope, A. Jones, C. Dearden, and T. J. Hinton, 2006: The physical properties of the atmosphere in the new Hadley Centre Global Environmental
Model (HadGEM1). Part I: Model description and global climatology. J. Climate, 19, 1274-1301.

McLandress, C., A. I. Jonsson, D. A. Plummer, M. C. Reader, J. F. Scinocca, and T. G. Shepherd, 2010: Separating the dynamical effects of climate change and ozone depletion. Part I: Southern Hemisphere stratosphere. J. Climate, 23, 5002-5020.

Orr, A., P. Bechtold, J. Scinocca, M. Ern, and M. Janiskova, 2010: Improved middle atmosphere climate and forecasts in the ECMWF model through a nonorographic gravity wave drag parameterization. J. Climate, 23, 5905-5926.

_ T. J. Bracegirdle, J. Scott Hosking, T. Jung, J. D. Haigh, T. Phillips, and W. Feng, 2012: Possible dynamical mechanisms for Southern Hemisphere climate change due to the ozone hole. J. Atmos. Sci., 69, 2917-2932.

Palmer, T. N., 1981: Diagnostic study of a wavenumber-2 stratospheric sudden warming in a transformed Eulerian-mean formalism. J. Atmos. Sci., 38, 844-855.

Randel, W. J., and F. Wu, 1999: Cooling of the Arctic and Antarctic polar stratospheres in response to ozone depletion. J. Climate, 12, 1467-1479.

Solomon, S., R. W. Portmann, T. Sasaki, D. J. Hofmann, and D. W. J. Thompson, 2005: Four decades of ozonesonde measurements over Antarctica. J. Geophys. Res., 110, D21311, doi:10.1029/2005JD005917.

Thompson, D. W. J., and S. Solomon, 2002: Interpretation of recent Southern Hemisphere climate change. Science, 296, 895-899.

Uppala, S. M., and Coauthors, 2005: The ERA-40 Re-Analysis. Quart. J. Roy. Meteor. Soc., 131, 2961-3012.

Wohltmann, I., and M. Rex, 2008: Improvement of vertical and residual velocities in pressure or hybrid sigma-pressure coordinates in analysis data in the stratosphere. Atmos. Chem. Phys., 8, 265-272.

WMO, 2007: Scientific assessment of ozone depletion: 2006. World Meteorological Organization Global Ozone Research and Monitoring Project Rep. 50, 572 pp. 